\title{
INTEGRATION OF "ON-CHIP" ELECTROCHEMICAL DETECTION IN A MICROFABRICATED CAPILLARY ELECTROPHORESIS DEVICE
}

\author{
Thomas J. Roussel, Jr ${ }^{1}$. and Robert S. Keynton \\ Department of Mechanical Engineering, University of Louisville \\ Louisville, KY 40292
}

\author{
Mark M. Crain, Vijay Bathlagundu, Douglas J. Jackson, \\ John A. Conklin, Rekha Pai, John F. Naber, \\ and Kevin M. Walsh \\ Department of Electrical and Computer Engineering, \\ University of Louisville \\ Louisville, KY 40292
}

\section{ABSTRACT}

The purpose of this effort has been to develop a selfcontained, transportable capillary electrophoresis (CE) with electrochemical (EC) detection analysis system, which contains all electrodes "on-chip" and employs specially made, miniaturized CE and EC supporting electronics. A novel fabrication process that produced recessed platinum electrodes was developed to form the $\mathrm{CE} / \mathrm{EC}$ device from two patterned ultra-flat glass substrates. The electrodes in the bottom substrate were formed by a self-aligned etch and deposition technique using a photholithographic lift-off process. The top substrate was etched to form the microchannels (20 $\mu \mathrm{m}$ deep x $50 \mu \mathrm{m}$ wide [avg]) and thermally bonded to the bottom substrate. The CE/EC experiments were performed using $0.02 \mathrm{M}$ phosphate buffer $(\mathrm{pH} 6)$ and a buffer/analyte solution $(2.2$ $\mathrm{mM}$ dopamine, $2.3 \mathrm{mM}$ catechol) with an applied voltage of $250 \mathrm{~V}$, which was delivered by a custom electronics unit interfaced to a laptop computer equipped with data acquisition software. Detection limits $(\mathrm{S} / \mathrm{N}=3)$ were found to be $4.3 \mu \mathrm{M}$ for dopamine and $4.4 \mu \mathrm{M}$ for catechol. A linear response was observed up to $2200 \mu \mathrm{M}$ and $2300 \mu \mathrm{M}$ for dopamine and catechol, respectively. Overall, the analytical performance of our CE/EC device is comparable to previously reported hybrid lab-on-a-chip devices with external $\mathrm{CE}$ and/or EC electrodes.

\section{INTRODUCTION}

Lab-on-a-chip detection schemes have most often utilized laser induced fluorescence (LIF) delivered in an off-chip format where the detection system itself is significantly larger than the chip. Electrochemical (EC) detection is more ideally suited to an on-chip format as demonstrated by an increasing number of capillary electrophoresis (CE) paired with EC detection applications for microchip devices [1-11]. However, in virtually all cases, both the EC detection electrodes crucial to sensitivity and selectivity and the $\mathrm{CE}$ driving electrodes have been conventional in size and design, consisting of manually positioned wire electrodes inserted into the chip. Our lab-on-a-chip efforts have been directed toward the development of self-contained and transportable CE/EC analysis systems that (a) incorporate all the required electrodes directly on the chip (Figure 1) and (b) utilize miniaturized $\mathrm{CE}$ and EC supporting electronics that are designed especially for the purpose of supporting our microchip platforms.

\author{
Jayadeep Gullapalli and Richard P. Baldwin \\ Department of Chemistry, \\ University of Louisville \\ Louisville, KY 40292
}

\section{FABRICATION}

A novel fabrication process that yielded recessed platinum electrodes was developed to form the CE/EC device from two photolithographically patterned ultra-flat glass substrates (precoated Soda Lime glass photomasks - Cr layer and AZ1518 photoresist). The electrodes on the bottom substrate were formed by creating $0.3 \mu \mathrm{m}$ recessions in the glass, using the existing chrome/photoresist layer as a masking layer with a self-aligned etch in a $6: 1 \mathrm{BOE}$ solution at $25^{\circ} \mathrm{C}$ for 30 seconds. This step was

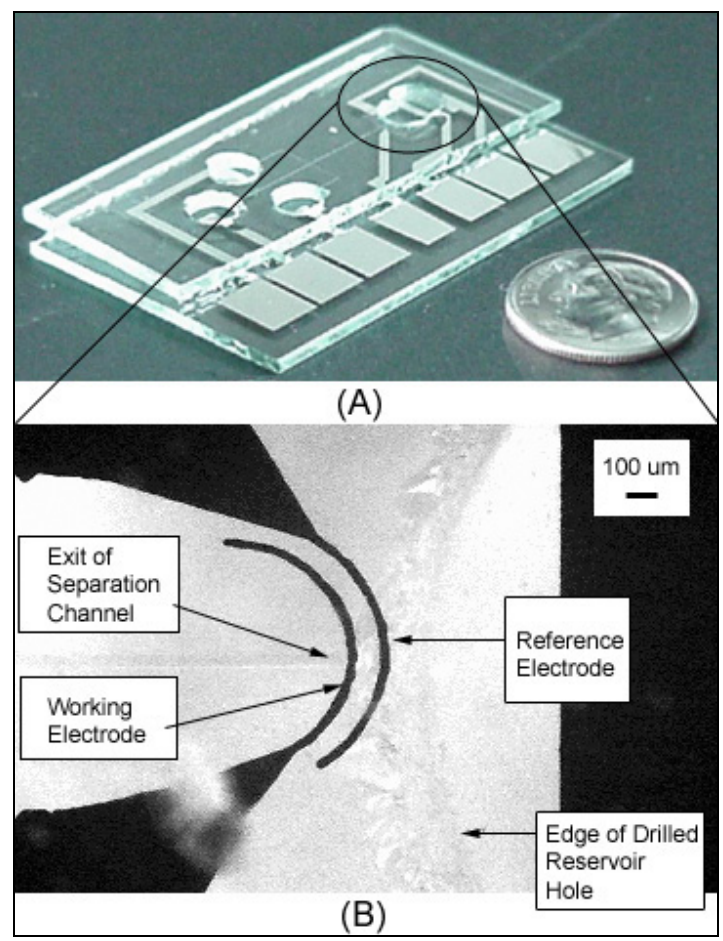

Figure 1. Photographs of (A) entire CE/EC microchip and (B) magnified (30x) top view of the EC cell and electrodes.

\footnotetext{
${ }^{1}$ "Travel support has been generously provided by the Transducers Research Foundation and by the DARPA MEMS and DARPA BioFlips programs."
} 
followed by sputter deposition of $\mathrm{Ti}$ as an adhesion layer $(\mathrm{t}=10$ $\mathrm{nm})$ followed by Platinum $(\mathrm{t}=300 \mathrm{~nm})$. The final electrode design was realized with a photolithographic liftoff process. The microchannels $(20 \mu \mathrm{m}$ deep x $50 \mu \mathrm{m}$ wide [average]) were created with wet chemical etching using a 6:1 BOE solution at room temperature for 30 minutes. The capillary system design consisted of a "balanced-cross", in that each arm was $1 \mathrm{~cm}$ in length with two arms bent at $90^{\circ}$ to facilitate placement on the $3.5 \mathrm{~cm} \times 5.0 \mathrm{~cm}$ final substrate. Subsequently, thermal fusion bonding was used to mate the two substrates and hermetically seal the capillary system. A $3^{\circ} \mathrm{C}$ per minute ramp up to and down from the bonding temperature of $620^{\circ} \mathrm{C}$ was critical to ensure that thermal stresses introduced into the glass substrates were not large enough to fracture either part. The slight recession of the electrodes beneath the surface of the glass dramatically affected the yield of the fabrication process by allowing a more conformal bond between the two glass surfaces. This step also aids in electrically isolating the electrode traces from one another. The strategic design and placement of the EC detection electrodes contributed strongly to the microchip performance.

\section{EXPERIMENTAL DETAILS}

$\mathrm{EC}$ and $\mathrm{CE}$ experiments were performed with $0.020 \mathrm{M} \mathrm{pH} 6.0$ phosphate buffer and a buffer/analyte solution sample $(2.2 \mathrm{mM}$ dopamine, $2.3 \mathrm{mM}$ catechol; Figure 2). An applied separation voltage of $250 \mathrm{~V}$ was delivered by a custom-made miniaturized electronics unit interfaced to a laptop computer equipped with both a control interface for the microchip system as well as data acquisition software for collecting EC detection data [12-14]. Amperometric EC detection was conducted with a three-electrode potentiostat included in the electronics designed for this system, which was powered by a single $9 \mathrm{~V}$ battery [14-15]. The sensitivity of the EC detection system was performed via testing with known concentrations of the samples described previously; then each sample was diluted to a lesser concentration and tested again. This was repeated until the sample could no longer be detected by the system.

Short-term (Figure 3) and long-term (Figure 4) repeatability and stability studies of our CE/EC devices were conducted. The short-term studies involved repeating the same set of experiments for an extended period of time, while holding all controllable factors (temperature, applied injection and separation voltages, EC potential voltage, etc.) constant. The volumes of solutions in the capillary-end reservoirs were maintained at a constant level because these small volumes $(\sim 150 \mu \mathrm{l})$ tend to evaporate fast, and evaporation could affect the repeatability data. In all cases, a series of experiments did not last long enough to deplete any reservoir of either buffer solution or sample. Additionally, electrolysis of the buffer solution, which usually leads to bubble generation and, in turn, experimental failure, was not observed. The long-term studies examined experimental data spread over days and weeks, comparing results from situations where the experimental factors were the same or similar.

\section{RESULTS}

The detection limit ( $\mathrm{S} / \mathrm{N}=3$ ) was found to be $4.3 \mu \mathrm{M}$ and 4.4 $\mu \mathrm{M}$ for dopamine and catechol, respectively, at an applied potential of $+0.75 \mathrm{~V}$ (vs. Pt). Linear responses up to $2200 \mu \mathrm{M}$ (rsquared $=0.9991)$ and $2300 \mu \mathrm{M}(\mathrm{r}$-squared $=0.9959)$ were observed for both dopamine and catechol, respectively. These

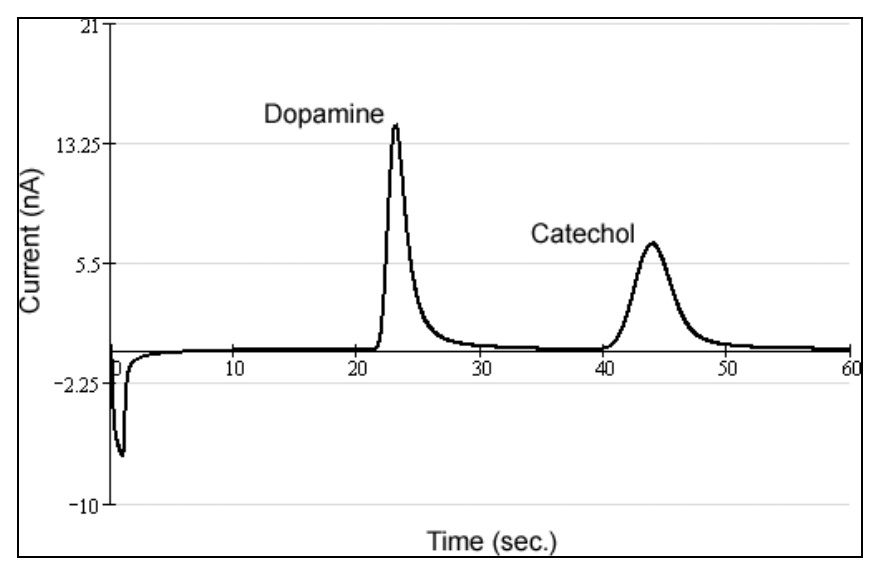

Figure 2. Sample electropherogram obtained for dopamine $(2.2$ $m M)$ and catechol $(2.3 \mathrm{mM})$ in $\mathrm{pH} 6$ phosphate buffer for "optimum" microchip configuration. Conditions: CE separation voltage $=250 \mathrm{~V} ; E C$ potential $=+0.75 \mathrm{~V}$ vs. Pt reference electrode.

findings are comparable to other studies that utilized nonintegrated electrodes $[6,10,11]$. As shown in Figure 3, the repeatability study showed nearly identical peak heights between successive experiments. These experiments were conducted without cleaning the system between successive injection/separations. The results suggest that the system injects a very precise and repeatable volume of sample $(\sim 125 \mathrm{pL})$. Additionally, any buildup of unreacted analyte in the detection reservoir can be neglected, at least over a handful of experiments, in that the unused sample in the detection reservoir did not appear to affect the results. The long-term stability tests demonstrated similar migration times and peak currents for dopamine throughout the time period investigated ( 8 months of studies) and the microchips included in the study show no signs of deterioration. Figure 4 represents a typical collection of results spanning roughly sixty days. In these experiments, new solutions of buffer and sample, with identical composition, were used. The plots indicate that the repeatability of the EC detection is consistent and highly predictable.

Several design characteristics were found to significantly affect the analytical performance of the on-chip EC electrodes: (1) the distance between the EC working electrode and the CE channel exit, which was directly responsible for the sensitivity and response of the EC detection circuitry; (2) the formation of a "shelf" restricting the height of the detection reservoir in the vicinity of the working electrode, which minimized the initial diffusion of the analyte plug upon exiting the separation channel; and, (3) the material and position of the reference electrode, which helps to accurately control the potential applied to the working electrode The peak current observed for dopamine was plotted as a function of the applied potential to generate a series of "hydrodynamic voltammograms" (Figure 5) for comparison with cyclic voltammetry data. These plots illustrate the EC detector response to a range of applied oxidation potentials and indicate optimal operating potential. In three-electrode potentiostats, the output of the working electrode will exhibit these unique 's'curves and are traditionally used to establish an optimal oxidation potential for the EC detection system with detection electrodes of a particular chemical makeup. These curves, however, are also 
influenced by the chemical makeup of the reference electrode. To explore this effect, the microfabricated $\mathrm{Pt}$ reference electrode was converted in situ to an $\mathrm{Ag} / \mathrm{AgCl}$ electrode to evaluate electrode material effects. As is shown in the changes between curves A and $\mathrm{B}$ in Figure 5, there is a noticeable and expected shift in the ' $\mathrm{s}$ 'curve after the in-situ modification, indicating that the modification to $\mathrm{Ag} / \mathrm{AgCl}$ was successful.

\section{CONCLUSIONS}

For the first time, traditional photolithographic processes have been used to incorporate both $\mathrm{CE}$ high voltage and $\mathrm{EC}$ detection electrodes directly onto the microchip platform. The analytical performance of the microfabricated devices, in both the hydrodynamic voltammogram results and the sensitivity of the system, is comparable to previously reported hybrid lab-on-a-chip devices with external $\mathrm{CE}$ and/or EC electrodes. The increasing demand for on-chip micro-systems to perform multiple analyses from a single sample injection has been corroborated by the shortterm results presented, enhancing the ability to do quantitative analyses with an ever-decreasing quantity of original sample. The long-term repeatability results are quite surprising, considering that some devices have undergone several hundreds of experiments and are still producing nearly identical results.

\section{ACKNOWLEDGEMENTS}

This work was supported by the National Science Foundation XYZ-on-a-chip program under grant \# CTS-9980831 and by the Department of Energy under grant \# 46411101095.

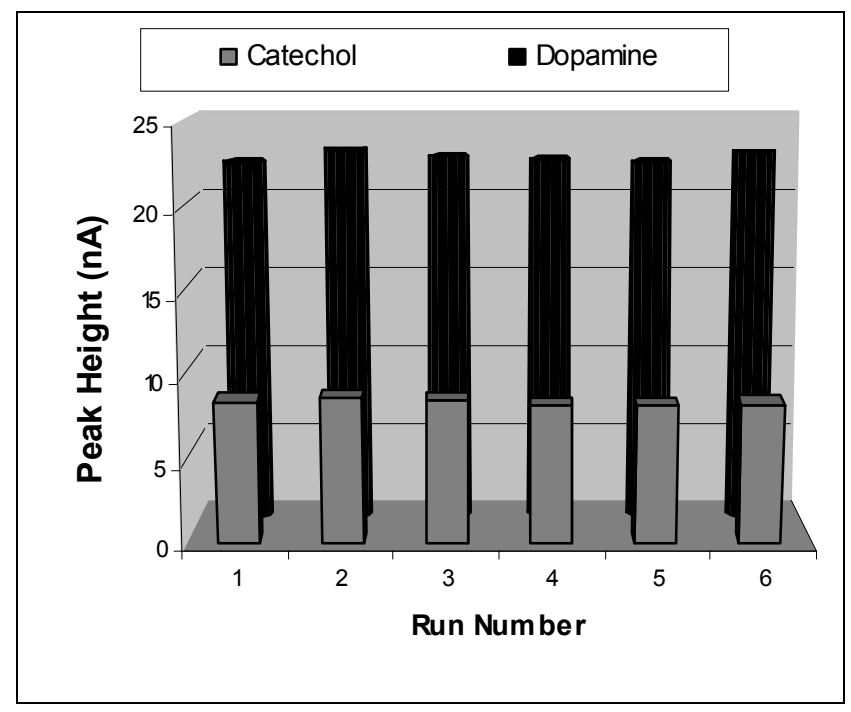

Figure 3. Dopamine and catechol peak currents for a series of 6 sequential injections. Conditions as in Figure 2.

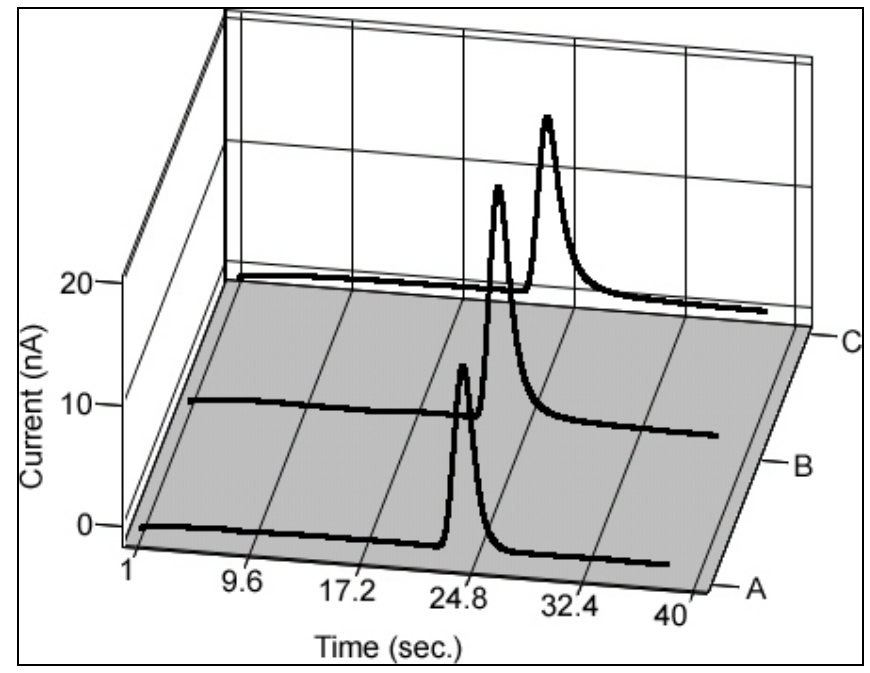

Figure 4. Electropherograms observed for dopamine $(2.2 \mathrm{mM})$ with same microchip device on (A) 7/18/01, (B) 9/18/01, and (C) 9/26/01. Conditions as in Figure 2.

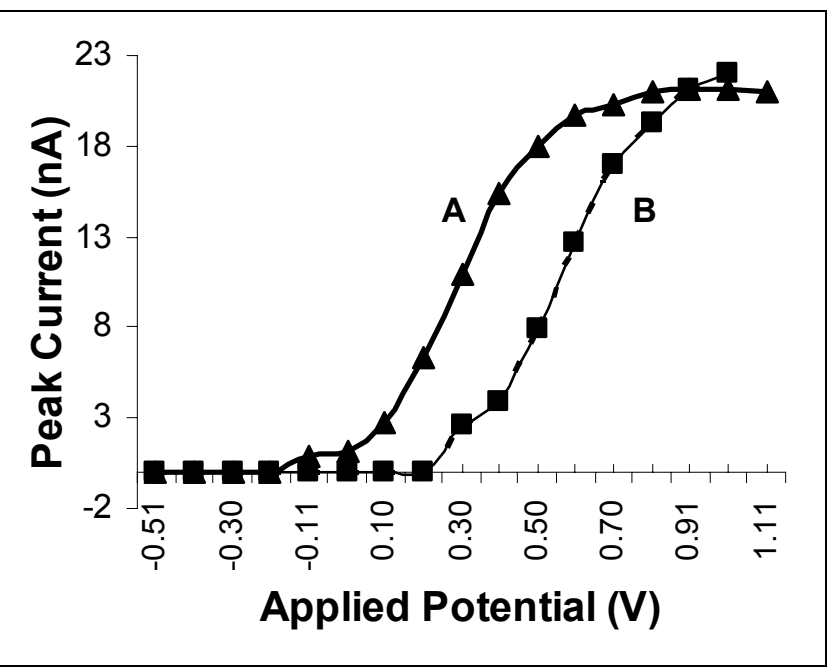

Figure 5. Two hydrodynamic voltammograms of (A) dopamine with Pt pseudo-reference electrode, and (B) dopamine with in-situ modified $\mathrm{Ag} / \mathrm{AgCl}$ reference electrode.

\section{REFERENCES}

1. J. Wang, B. Tian, and E. Sahlin, "Integrated Electrophoresis Chips/Amperometric Detection with Sputtered Gold Working Electrodes", Analytical Chemistry, 1999, 71(17), pp. 3901-3904. 
2. J. Wang, B. Tian, and E. Sahlin, "Micromachined Electrophoresis Chips with Thick-Film Electrochemical Detectors", Analytical Chemistry, 1999, 71(23), pp. 5436-5440.

3. J. Wang, M. P. Chatrathi, and B. Tian, "Microseparation Chips for Performing Multienzymatic Dehydrogenase/Oxidase Assays: Simultaneous Electrochemical Measurement of Ethanol and Glucose", Analytical Chemistry, 2001, 73(6), pp. 1296-1300.

4. J. Wang, M. P. Chatrathi, A. Mulchandani and W. Chen, "Capillary Electrophoresis Microchips for Separation and Detection of Organophosphate Nerve Agents", Analytical Chemistry, 2001, 73(8), pp. 1804-1808.

5. C. S. Henry, M. Zhong, S. M. Lunte, M. Kim, H. Bau, and J. J. Santiago, "Ceramic Microchips for Capillary ElectrophoresisElectrochemistry”, Anal. Commun., 1999, 36, pp. 305-307.

6. R. S. Martin, A. J. Gawron, S. M. Lunte, and C. S. Henry, "Dual-Electrode Electrochemical Detection for Poly(dimethylsiloxane)-Fabricated Capillary Electrophoresis Microchips", Analytical Chemistry, 2000, 72(14), pp. 3196-3202.

7. A. J. Gawron, R. S. Martin, and S. M. Lunte, "Fabrication And Evaluation of a Carbon-Based Dual-Electrode Detector for Poly(dimethylsiloxane) Electrophoresis Chips", Electrophoresis, 2001, 22, pp. 242-248.

8. J. S. Rossier, M. A. Roberts, R. Ferrigno, and H. H. Girault, "Electrochemical Detection in Polymer Microchannels", Analytical Chemistry, 1999, 71(19), pp. 4294-4299.

9. J. S. Rossier, A. Schwarz, F. Reymond, R. Ferrigno, F. Bianchi, and H. H. Girault, "Microchannel Networks for Electrophoretic Separations", Electrophoresis, 1999, 20, pp. 727731.

10. J. S. Rossier, R. Ferrigno, and H. H. Girault, "Electrophoresis with Electrochemical Detection in a Polymer Microdevice", Journal of Electroanalytical Chemistry, 2000, 492 (1), pp. 15 - 22.

11. M. A. Schwarz, B. Galliker, K. Fluri, T. Kappes, and P. C. Hauser, "A Two-Electrode Configuration for Simplified Amperometric Detection in a Microfabricated Electrophoretic Separation Device", Analyst, 2001, 126, pp. 147-151.

12. D. J. Jackson, J. F. Naber, T. J. Roussel, Jr., V. Bathlagundu, M. M. Crain, R. Pai, J. A. Conklin, R. P. Baldwin, R. S. Keynton, and K. M. Walsh, manuscript in preparation for Analytical Chemistry.

13. D. Jackson, J. Naber, R. Baldwin, K. Walsh, R. Keynton, M. Crain, T. Roussel, and M. Diaconu, "A Compact Battery Powered High Voltage Power Supply for Capillary Electrophoresis Using Microchip Capillaries", Proceedings of the Pittsburgh Conference on Analytical Chemistry and Applied Spectroscopy (PITTCON 2001), \#116, 2001.

14. V. Bathlagundu, D. Jackson, J. Naber, R. Baldwin, K. Walsh, R. Keynton, M. Crain, T. Roussel, M. Diaconu, "A Portable Battery Powered Electrochemical Detection Circuit With a 1000 Volt CE Power Supply for Microchip Capillaries", Proceedings of the Fourteenth Biennial University/ Government/ Industry Microelectronics Symposium, pp. 197-200, 2001.
15. D. Jackson, J. Naber, R. Baldwin, K. Walsh, R. Keynton, M. Crain, T. Roussel, and M. Diaconu, "A Portable Battery Powered Electrochemical Detection Circuit", Proceedings of the Pittsburgh Conference on Analytical Chemistry and Applied Spectroscopy (PITTCON 2001),\#1345, 2001. 